\title{
Angiomyofibroblastoma of the vulva diagnosed preoperatively: A case report
}

\author{
TADAHIRO SHOJI ${ }^{1}$, RYOSUKE TAKESHITA $^{1}$, RIKA MUKAIDA ${ }^{1}$, \\ TAMOTSU SATO $^{1}$, MASAMI TAGUCHI ${ }^{2}$ and SHUNICHI SASOU ${ }^{3}$
}

\author{
Departments of ${ }^{1}$ Obstetrics and Gynecology, ${ }^{2}$ Radiology and ${ }^{3}$ Pathology and Laboratory \\ Medicine, Hachinohe Red Cross Hospital, Hachinohe, Aomori 039-1104, Japan
}

Received March 29, 2017; Accepted June 13, 2017

DOI: $10.3892 / \mathrm{mco} .2017 .1347$

\begin{abstract}
A 50-year-old woman (gravida 2, para 2) first noticed a small nodule in the right labium majus 2 years prior to the initial visit to the Hachinohe Red Cross Hospital (Hachinohe, Japan), which had gradually increased in size. On physical examination, a well-circumscribed, elastic, firm, goose egg-sized, subcutaneous mass protruding from the right labium majus was identified. On magnetic resonance imaging (MRI), the lesion was hypointense on T1-weighted images and was well-circumscribed, strongly enhanced and homogeneous on gadolinium-enhanced images, measuring $7.5 \times 4 \mathrm{~cm}$. The same tumor had measured $2.6 \mathrm{~cm}$ on an MRI performed 6 years earlier. Based on the clinical course and imaging findings, angiomyofibroblastoma was diagnosed and surgical resection of the tumor was performed. The tumor was well-circumscribed and highly vascular. The intraoperative blood loss was $70 \mathrm{ml}$. Histopathologically, the tumor cells were concentrated around blood vessels, were spindle-shaped to oval with mild atypia, and were positive for vimentin, desmin, neural cell adhesion molecule (N-CAM), CD-34, estrogen receptor and progesterone receptor, and negative for S-100. Based on these findings, the diagnosis of angiomyofibroblastoma was confirmed. Angiomyofibroblastoma is a benign mesenchymal tumor that occurs in the female external genitalia. Differentiation of this tumor from aggressive angiomyxoma, a fast-growing infiltrative malignancy that occurs in the same region, may be challenging. The diagnosis of angiomyofibroblastoma is usually based on the histopathological findings of the resected specimen. The present case is of value, as the angiomyofibroblastoma was successfully diagnosed preoperatively based on the clinical course and imaging findings.
\end{abstract}

Correspondence to: Dr Tadahiro Shoji, Department of Obstetrics and Gynecology, Hachinohe Red Cross Hospital, Tamonoki Nakaaketo 2, Hachinohe, Aomori 039-1104, Japan

E-mail: tshoji@iwate-med.ac.jp

Key words: vulva tumor, soft tissue neoplasm, angiomyofibroblastoma, aggressive angiomyxoma

\section{Introduction}

Angiomyofibroblastoma (AMFB) is a benign mesenchymal soft tissue tumor first described by Fletcher et al in 1992 (1). AMFB commonly develops in the female external genitalia, but it has also been reported to occur in the male scrotum or groin $(2,3)$. The average age at onset is in the late 30 s, and it is a well-circumscribed, elastic, soft tumor, usually sized $<5 \mathrm{~cm}$, white to yellowish brown and sponge-like or myxoid on cross-section. Histologically, the tumor cells are oval to spindle-shaped and arranged in a fascicular, wavy or palisade pattern, with areas of high and low cell density, with stromal proliferation of small vessels. Immunohistochemically, the tumor cells are usually positive for vimentin, desmin, estrogen receptor and progesterone receptor, and negative for S-100 and $\alpha$-smooth muscle actin ( $\alpha$-SMA), although desmin-negative and $\alpha$-SMA-positive cases have been reported (1,3-5). AMFB may be difficult to distinguish from aggressive angiomyxoma (AAM). AMFB is usually diagnosed based on the histopathological findings of the resected specimens. The aim of the present study was to report an AMFB case that was diagnosed preoperatively based on the clinical course and magnetic resonance imaging (MRI) findings and describe our experience with the management of this patient, along with a review of the related literature.

\section{Case report}

A 50-year-old woman (gravida 2, para 2) presented with the chief complaint of a palpable mass in the vulva. The patient's family history was unremarkable, but she had been diagnosed with uterine adenomyosis at the age of 44 years and underwent abdominal total hysterectomy. Two years earlier, the patient had noticed the presence of a small nodule in the right labium majus, but it was left untreated. The nodule gradually increased in size and was eventually accompanied by spontaneous pain, prompting the patient to visit our department. At the initial visit, an elastic, firm, goose egg-sized subcutaneous mass protruding from the right labium majus was detected on physical examination (Fig. 1). On palpation, the mass was well-circumscribed, with good mobility against the surrounding tissues. The skin overlaying the mass was macroscopically normal, and no subjective 
pain was noted. There were no abnormalities in the complete blood count or biochemical examinations. The tumor marker levels of carcinoembryonic antigen $(1.0 \mathrm{ng} / \mathrm{ml})$, carbohydrate antigen (CA)19-9 (<2.0 U/ml) and CA $125(8.8 \mathrm{U} / \mathrm{ml})$, were also normal. MRI scans revealed a solid tumor measuring $7 \mathrm{~cm}$ in diameter under the skin on the right labium majus, which was sharply marginated, without infiltration of the deep tissues. The tumor was hypointense on T1-weighted images and exhibited mostly homogeneous enhancement on fat-saturated (fat-sat) gadolinium-enhanced images, although there was a poorly enhanced area at the center, which was attributed to either necrosis or degeneration (Fig. 2A and B). The patient had previously undergone fat-sat T2-weighted MRI prior to undergoing hysterectomy at the age of 44 years, which detected a mass measuring $2.6 \mathrm{~cm}$ in the same region (Fig. 2C). Based on the clinical course and imaging findings, AMFB was diagnosed and surgery was performed. The tumor was removed under general anesthesia. Although the tumor was well-circumscribed, it was highly vascular, and the intraoperative ligation of the blood vessels was time-consuming. The operative time was $41 \mathrm{~min}$ and the intraoperative blood loss was $70 \mathrm{ml}$. The postoperative course was eventless and the patient was discharged on the 7 th postoperative day. Macroscopically, the resected tumor measured $8 \times 7 \times 5 \mathrm{~cm}$. The tumor was not encapsulated, but was well-circumscribed, white, edematous and solid (Fig. 3A and B). Histologically, the tumor consisted of sparse collagen fibers, along with small to medium-sized blood vessels, with a scattered or clustered distribution. The tumor cells were aggregated around blood vessels, they were oval to spindle-shaped and had acidophilic and fibrous cytoplasm. Outside the perivascular area, cellular components were sparse. No mucin deposition was seen in the stroma, and there was no thickening or hyalinization of the vascular walls (Fig. 4). On immunohistochemical examination, the tumor cells were positive for vimentin, desmin, $\mathrm{N}-\mathrm{CAM}, \mathrm{CD}-34$, estrogen receptor and progesterone receptor, and negative for S-100. The Azan-Mallory staining result was also positive (Fig. 5A-H). Based on these findings, the histopathological diagnosis of AMFB was confirmed. At 10 months postoperatively, no recurrence was observed.

Written informed consent was obtained from the patient regarding the publication of the case details and associated images.

\section{Discussion}

AMFB is a benign mesenchymal soft tissue tumor first described by Fletcher et al in 1992 (1). AMFB commonly develops in the female external genitalia, but there have also been a few reports of AMFB involving the male scrotum or groin $(2,3)$. As regards the clinical characteristics of AMFB, the average age at onset is in the late $30 \mathrm{~s}$, and it is a well-circumscribed, elastic, soft subcutaneous tumor, usually $<5 \mathrm{~cm}$ in diameter; the cut surface appears white to yellowish brown and sponge-like or myxoid. Histologically, oval to spindle-shaped tumor cells are arranged in a fascicular, wavy or palisade pattern, with areas of both high and low cell density. Proliferation of small vessels is noted in the stroma. Immunohistochemically, the tumor cells are usually positive for vimentin, desmin, estrogen receptor and progesterone receptor, and negative for S-100 and

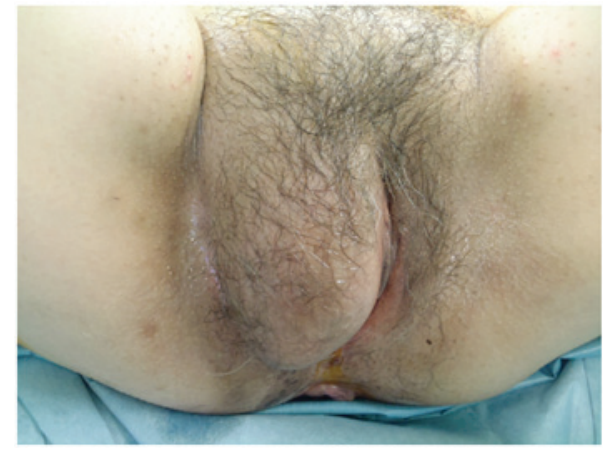

Figure 1. On physical examination, an elastic, firm, well-circumscribed, goose egg-sized subcutaneous tumor was identified protruding from the right labium majus.

$\alpha$-SMA, although some cases are reportedly desmin-negative and $\alpha$-SMA-positive (1,3-5).

It is important to be aware that AAM must be considered in the differential diagnosis of AMFB. AAM was described in 1983 by Steeper and Rosai (6). AAM is a tumor of the external genitalia that affects young women and is similar to AMFB in terms of both clinical presentation and histopathological characteristics. AAM grows rapidly and infiltrates the surrounding tissues. This tumor often recurs locally after surgical resection, but usually does not metastasize (6). As regards the histological differences between AMFB and AAM, the former contains both hypo- and hypercellular areas, whereas the latter exhibits sparse, homogeneous growth of tumor cells with atypical nuclei. While the stroma in AAM displays mucin deposition, proliferation of small to medium-sized blood vessels, dilation of vascular spaces, hyalinization and thickening of vascular walls, the stroma in AMFB only exhibits proliferation of small blood vessels. On immunohistochemical examination, the tumor cells of both AAM and AMFB are positive for vimentin, estrogen receptor and progesterone receptor and negative for $\mathrm{S}-100$, but the cell kinetics for desmin and $\alpha$-SMA may differ among individual cases. Therefore, immunochemical results alone are not sufficient to distinguish AMFB from AAM. AAM grows rapidly and reaches a diameter of $>5 \mathrm{~cm}$. In the majority of $\mathrm{AMFB}$ cases, the tumor is sized $<5 \mathrm{~cm}$ (7). According to Nagai et al, the average diameter of AMFB is $4.5 \mathrm{~cm}$ (7). However, there are reports describing AMFB cases with tumors $>10 \mathrm{~cm}$ in size (8), and it is critical to distinguish these cases from AAM. The imaging characteristics that differentiate AMFB from AAM are listed in Table I. While AMFB appears well-circumscribed with strong heterogeneous enhancement, AAM has a strong infiltrative tendency and is poorly circumscribed, while the contrast-enhanced images are characterized by a whorled or swirling growth pattern $(9,10)$.

In the present case, based on the imaging characteristics and clinical course, the preoperative diagnosis was AMFB and surgery was performed. The tumor was $8 \mathrm{~cm}$ in diameter, which was rather large for an AMFB. Reducing intraoperative blood loss is important, and prior studies have explored measures to prevent excessive bleeding. Quintero et al reported that they succeeded in reducing blood loss in a patient with a large AMFB, $12 \mathrm{~cm}$ in diameter, by performing preoperative embolization (11). In the present case, there were several 
Table I. Differentiation between AMFB and AAM.

\begin{tabular}{lll}
\hline Characteristics & \multicolumn{1}{c}{ AMFB } & \multicolumn{1}{c}{ AAM } \\
\hline Imaging findings & Well-circumscribed & Infiltrative tendency \\
& Strong heterogeneous enhancement & Enhanced in a whorled or swirling growth pattern \\
& Relatively small $(\leq 5 \mathrm{~cm})$ & Large $(\geq 5 \mathrm{~cm})$ \\
Pathological findings & Oval to spindle-shaped cells & Stellate to spindle-shaped cells \\
& Both hyper- and hypocellular areas are present & Sparse, homogeneous cell distribution \\
Clinical outcome & Recurrence is rare & Recurrence is frequent \\
\hline
\end{tabular}

AMFB, angiomyofibroblastoma; AAM, aggressive angiomyxoma.

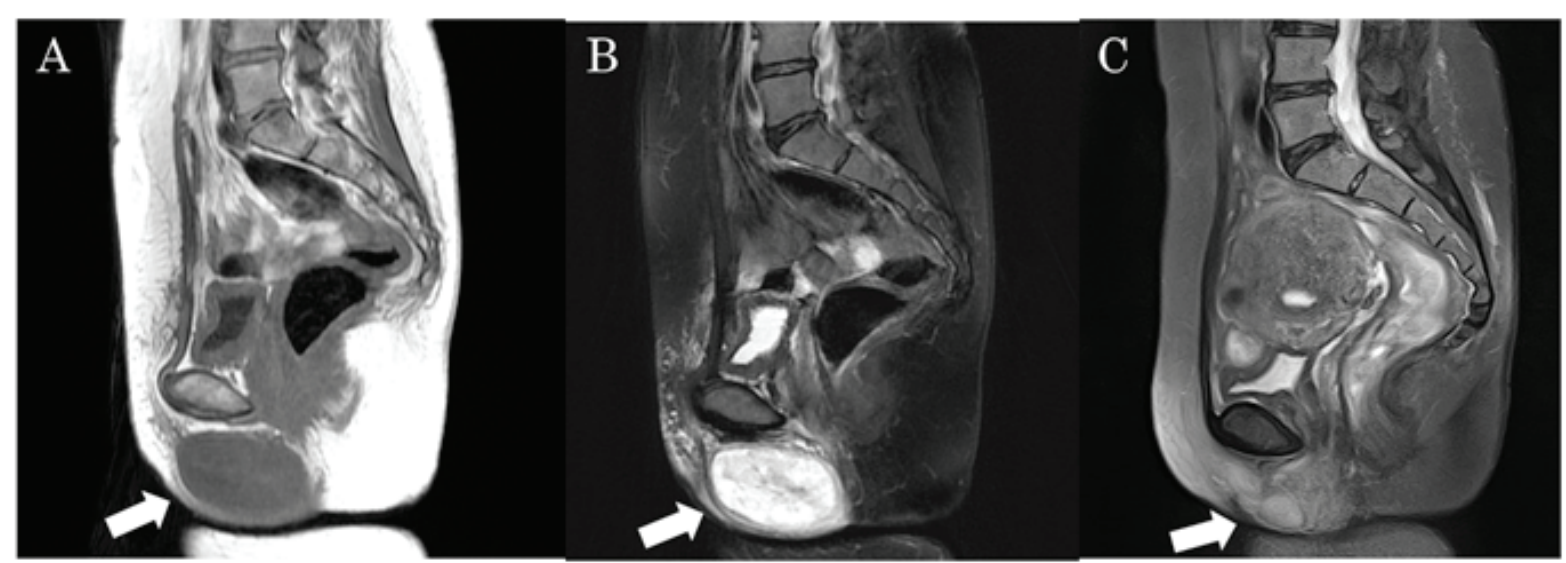

Figure 2. On magnetic resonance imaging (MRI), a well-circumscribed mass measuring $7.5 \times 4 \mathrm{~cm}$ was identified under the skin of the right labium majus. (A) The mass appeared contrast-enhanced on fat-saturated (fat-sat) gadolinium-enhanced images and (B) hypointense on T1-weighted images. (C) MRI findings from 6 years earlier: Fat-sat T2-weighted images revealed a well-circumscribed mass measuring $2.6 \times 1.7 \mathrm{~cm}$ that appeared mildly hyperintense.
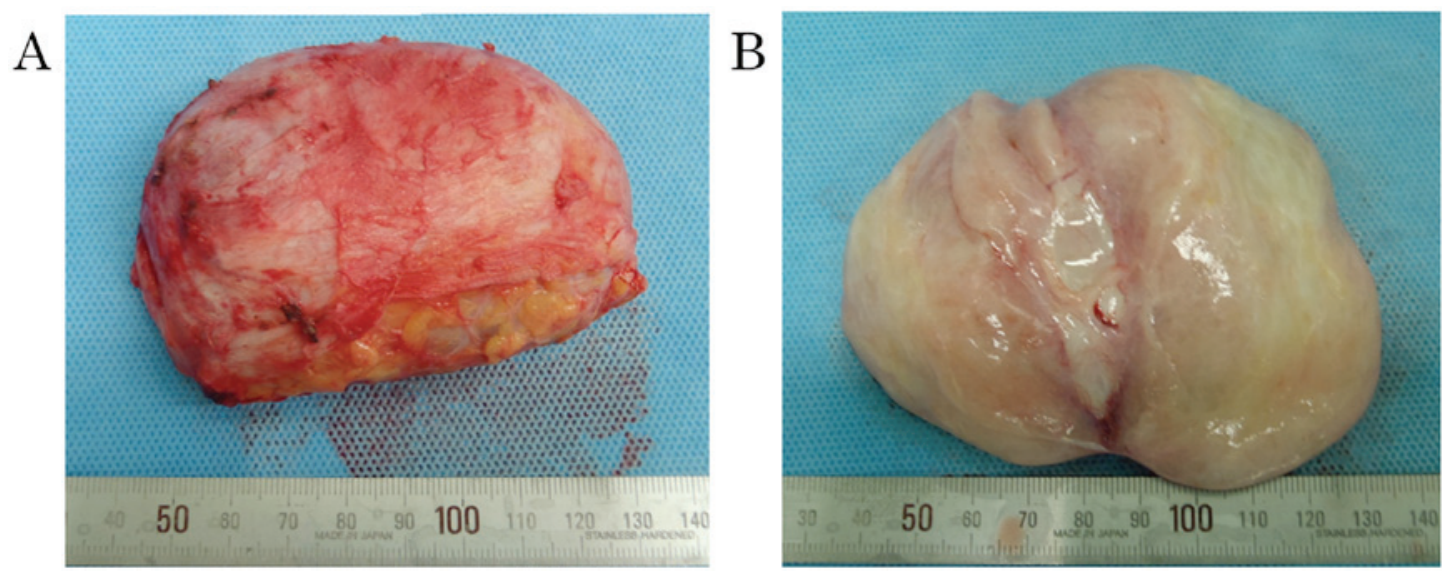

Figure 3. (A) The resected tumor measured 8x7x5 cm, was non-encapsulated but well-circumscribed. (B) On cross section the tumor appeared white, edematous and solid.

blood vessels feeding the tumor, necessitating intraoperative ligation. Thus, the operation lasted longer than expected and the amount of blood loss was $70 \mathrm{ml}$. AMFB has a benign prognosis following simple surgical resection. However, in patients with large AMFBs, the blood vessels feeding the tumor are well-developed, and it is important to consider the possibility of excessive blood loss when performing surgery in such patients.
There are sporadic reports on using gonadotropin-releasing hormone $(\mathrm{GnRH})$ analogs for the preoperative reduction of AAM and prevention of postoperative recurrence (12-15). However, there have been no reports of their use for the treatment of AMFB. Thus, as there is no scientific evidence showing that $\mathrm{GnRH}$ analogs reduce the recurrence rate of AMFB, this patient is currently followed up without administering additional treatment after surgery. 


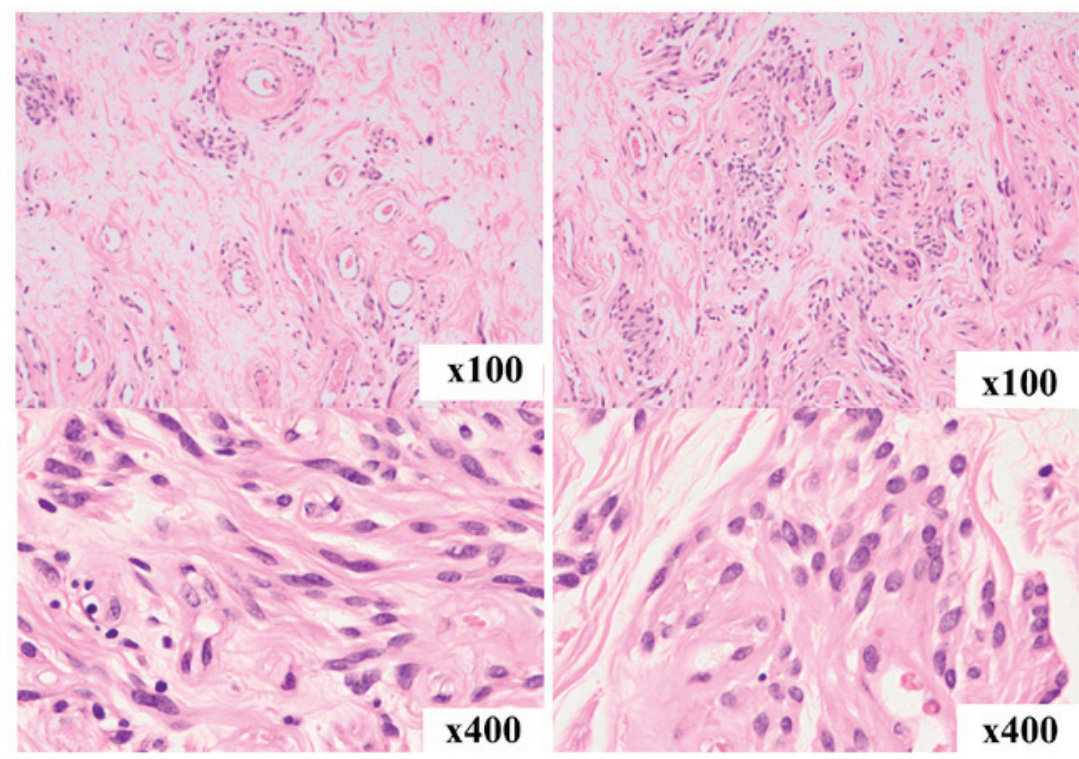

Figure 4. Histopathological findings. The tumor consisted of sparse collagen fibers and exhibited a higher cellular density around blood vessels. The cells proliferating around blood vessels were oval to spindle-shaped and had acidophilic and fibrous cytoplasm. No mucin deposition was seen in the stroma, and there was no thickening or hyalinization of vascular walls.
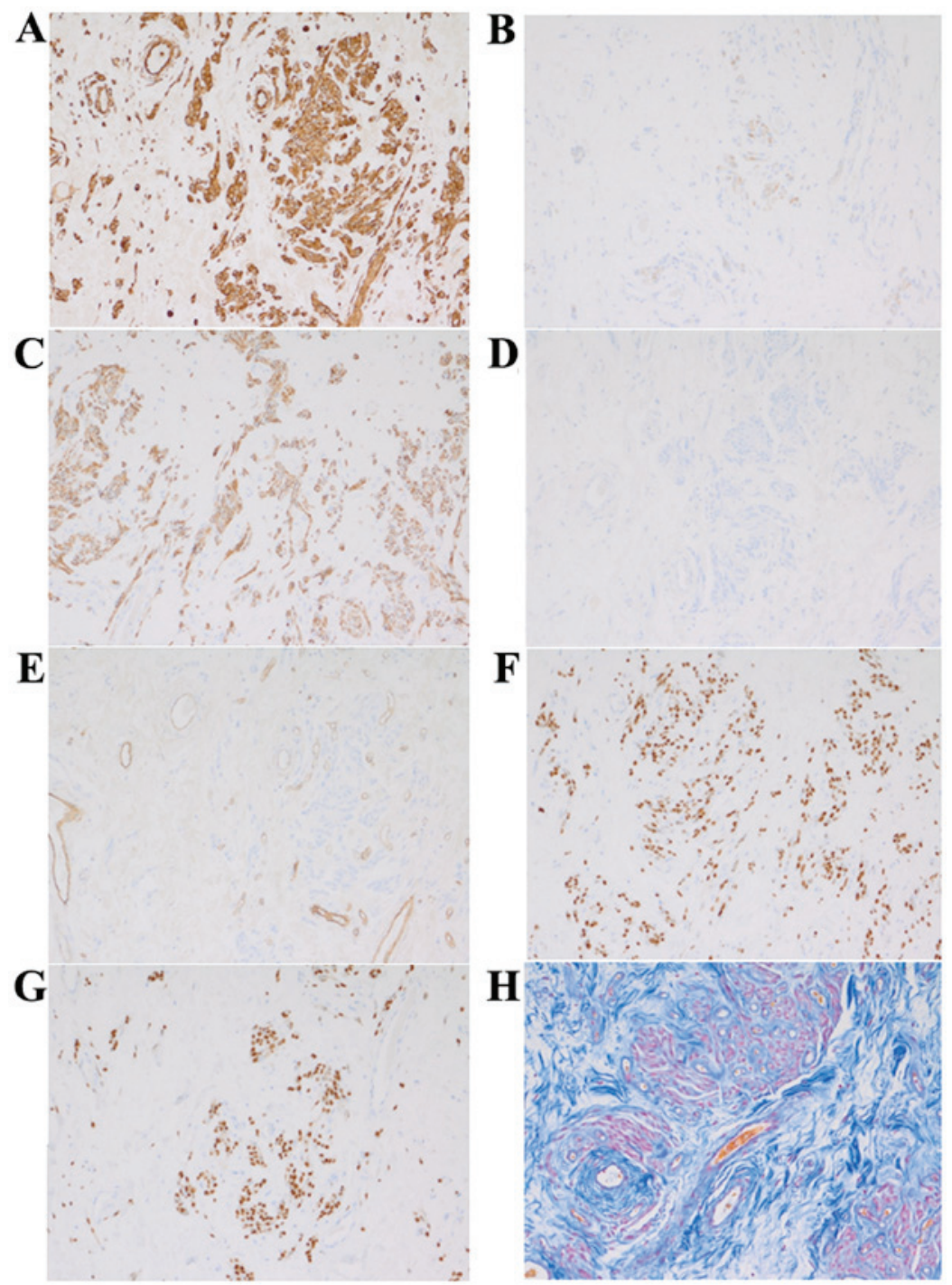

Figure 5. Immunohistochemistry and Azan-Mallory staining. (A) Vimentin, (B) desmin, (C) neural cell adhesion molecule, (D) S-100, (E) CD34, (F) estrogen receptor, $(\mathrm{G})$ progesterone receptor, (H) Azan-Mallory stain. 
The patient in the present case had also been diagnosed with uterine adenomyosis at the age of 44 years and underwent hysterectomy. The preoperative MRI performed at the time revealed a mass measuring $2.6 \mathrm{~cm}$ in diameter, but the patient received no medical treatment. When surgery was performed for the AMFB, the tumor was found to have grown to $7.5 \mathrm{~cm}$ in diameter over the previous 6 years, suggesting a relatively slow growth rate. Slow tumor growth is another characteristic of AMFB that distinguishes it from AAM, and was also one of the factors that facilitated the preoperative diagnosis. None of the past studies on AMFB used images obtained over the 6-year period prior to surgery for making comparisons. This report is considered valuable as it may serve as a verification of the relatively slow growth of AMFB.

\section{References}

1. Fletcher CD, Tsang WY, Fisher C, Lee KC and Chan JK: Angiomyofibroblastoma of the vulva. A benign neoplasm distinct from aggressive angiomyxoma. Am J Surg Pathol 16: 373-382, 1992.

2. Ding G, Yu Y, Jin M, Xu J and Zhang Z: Angiomyofibroblastomalike tumor of the scrotum: A case report and literature review. Oncol Lett 7: 435-438, 2014.

3. Ito M, Yamaoka H, Sano K and Hotchi M: Angiomyofibroblastoma of the male inguinal region. Arch Pathol Lab Med 124: 1679-1681, 2000.

4. Laskin WB, Fetsch JF and Tavassoli FA: Angiomyofibroblastoma of the female genital tract: Analysis of 17 cases including a lipomatous variant. Hum Pathol 28: 1046-1055, 1997.

5. Aono K, Sekine M, Serikawa T, Tojo Y, Hanaoka J and Takeuchi Y: Two cases of rare vulvar mesenchymal tumor: Comparisons of aggressive angiomyxoma with angiomyofibroblastoma. Acta Obst Gynaec Jpn 50: 975-978, 1998 (In Japanese).
6. Steeper TA and Rosai J: Aggressive angiomyxoma of the female pelvis and perineum. Report of nine cases of distinctive type of gynecologic soft-tissue neoplasm. Am J Surg Pathol 7: 463-475, 1983.

7. Nagai K, Adachi K and Saito H: Huge pedunculated angiomyofibroblastoma of vulva. Int J Clin Oncol 15: 201-205, 2010.

8. Qiu P, Wang Z, Li Y and Cui G: Giant pelvic angiomyofibroblastoma: Case report and literature review. Diagn Pathol 9: 106, 2014.

9. Sharon WW and Goldkum JR (eds): Enzinger \& Weiss's Soft Tissue Tumors 5th edition. Philadelphia, Mosby Elsevier, pp-1087, 2008.

10. Geng J, Hu S and Wang F: Large paravaginal angiomyofibroblastoma: Magnetic resonance imaging findings. Jpn J Radiol 29: 152-155, 2011.

11. Quintero C, Sasken H, Houck KL and Hernandez E: Angiomyofibroblastoma of the retroperitoneum: A case report. J Reprod Med 52: 741-744, 2007.

12. Lee CW, Yoon JH, Park DC and Lee SJ: Aggressive angiomyxoma of the vulva treated by using a gonadotropin-releasing hormone agonist: A case report. Eur J Gynaecol Oncol 32: 686-688, 2011.

13. Palomba S, Oppedisano R, Annunziata G, Zullo F and Amorosi A: Leuprolide acetate depot plus high-dose raloxifene hydrochloride before and after surgery for recurrent vaginal aggressive angiomyxoma: A case report. Gynecol Oncol 123: 172-173, 2011.

14. McCluggage WG, Jamieson T, Dobbs SP and Grey A: Aggressive angiomyxoma of the vulva: Dramatic response to gonadotropin-releasing hormone agonist therapy. Gynecol Oncol 100: 623-625, 2006.

15. Fine BA, Munoz AK, Litz CE and Gershenson DM: Primary medical management of recurrent aggressive angiomyxoma of the vulva with a gonadotropin-releasing hormone agonist. Gynecol Oncol 81: 120-122, 2001. 